\title{
Cuidar a una persona con trauma craneoencefálico, la experiencia desde los estudiantes
}

\author{
Providing care to a person with traumatic brain injury, the experience from the students' \\ standpoint
}

\section{Cuidar de uma pessoa com traumatismo crânio-encefálico, a experiência dos alunos}

\author{
Amalia Priscila Peña-Pita* \\ Emilce Salamanca-Ramos ${ }^{* *}$ \\ Mery Luz Valderrama-Sanabria ${ }^{* * *}$
}

\section{Resumen}

\begin{abstract}
Introducción: A nivel mundial los traumatismos son un problema de salud pública, dejando secuelas físicas, emocionales y psicológicas. El papel del profesional de enfermería es fundamental para disminuir las complicaciones y ofrecer un cuidado integral, asegurando una atención de calidad. Las experiencias de cuidado de los estudiantes son de gran utilidad, que dejan grandes enseñanzas, no solo en busca de un fin científico, sino también para determinar la sensibilidad y responsabilidad hacia el sujeto de cuidado. La investigación tuvo como objetivo, describir la experiencia de los estudiantes de enfermería en el cuidado de pacientes hospitalizados con trauma craneoencefálico. Materiales y Método: Investigación cualitativa de carácter fenomenológico. El muestreo se realizó por conveniencia, pertinencia, adecuación, oportunidad y disponibilidad. Se utilizaron como instrumentos la entrevista a profundidad y el diario de campo. Resultados: Se identificaron las categorías: "Me enfrento con la realidad", "Cuidado integral, es lo que siempre me han inculcado", "Mi relación con el otro", "Enseñándole a mi paciente y su familia”, y "La Familia, pilar fundamental". Conclusiones: La experiencia expresada por los estudiantes generó expectativas inesperadas, pero se aplicaron los conocimientos necesarios para brindar un mejor cuidado individualizado. En cada participante emergió la sensibilidad, lo que le permitió situarse en la posición del otro, comprendiendo la impotencia, frustración y pérdida de control. El proceso de Atención de Enfermería es el esquema necesario para brindar el cuidado integral del paciente.
\end{abstract}

Palabras clave: Educación en enfermería, estudiantes de enfermería, enfermería, cuidado.
Autor de correspondencia

* Enfermera. Magister en Educación. Magister en enfermería. Docente Universidad de los Llanos, Grupo de investigación GESI. Correo: prisicla.pena@unillanos.edu.co. Orcid: https://orcid.org/0000-0003-19473812. Villavicencio, Colombia.

** Magister en Enfermería. Candidata Doctorado en Ciencias Sociales, Niñez y Juventud. Docente Universidad de los Llanos, Grupo de investigación GESI. Correo: esalamanca@unillanos.edu.co. Orcid: https://orcid.org/0000-0002-3549-7067. Villavicencio, Colombia.

*** Enfermera. Magister en Enfermería. Especialista en Cuidado Crítico Pediátrico. Doctoranda en Educación. Docente Universidad de los Llanos, Grupo de Investigación de Cuidado. Villavicencio. Correo: mvalderrama@unillanos.edu.co. Orcid: https://orcid.org/0000-0003-16186009. Villavicencio, Colombia.

\begin{abstract}
Introduction: Globally, injury or physical trauma are a public health problem, leaving physical, emotional and psychological consequences. The role of the nursing professional is fundamental to reduce the complications and offer a comprehensive care, ensuring quality health care. The care experiences from the students are useful, leaving significant lessons, not only with a scientific end, but also to determine the sensibility and responsibility towards the patient. The objective of this research was
\end{abstract}

Este es un artículo bajo la licencia CC BY (https://creativecommons.org/ licenses/by/4.0/) @) (1) 
ISSN-PRINT

1794-9831

E-ISSN 2322-7028

Vol. 16 No. 3

Sep - Dic 2019

Cúcuta, Colombia

to describe the experience of the nursing students toward the care provided to hospitalized patients with traumatic brain injury. Materials and method: Qualitative research of phenomenological approach. The sampling was made by convenience, pertinence, adequation, opportunity and availability. Instruments such as in-depth interviews and a field diary were used. Results: The following categories were identified: "I face reality", "Comprehensive care, is what they always have taught me", "My relationship with the other", "Teaching my patient and their family", and "Family, a fundamental pillar". Conclusions: The experience shown by the students generated unforeseen expectations, but the necessary knowledge was applied to provide better individualized care. Each participant had sensibility, which allowed them to consider the position of the other person, understanding impotence, frustration and loss of control. The Nursing Care process is a scheme that is necessary to provide comprehensive care to the patient.

Keywords: Nursing education, nursing students, nursing, care.

\section{Resumo}

Introdução: Mundialmente os traumatismos são um problema de saúde pública, causando consequências físicas, emocionais e psicológicas. O papel do profissional de enfermagem é fundamental para reduzir as complicações e prestar cuidado integral, garantindo o atendimento de qualidade. As experiências do cuidado dos alunos são de muita utilidade, deixando ensinamentos, não só a procura de conhecimentos científicos, também para determinar a sensibilidade e responsabilidade com o sujeito de cuidado. Este estudo teve como objetivo, descrever a experiência de acadêmicos de enfermagem no cuidado de pacientes com traumatismo crânio-encefálico. Materiais e Métodos: Pesquisa qualitativa de caráter fenomenológico. A amostragem realizou-se por conveniência, pertinência, adequação, oportunidade e disponibilidade. Utilizaram-se como instrumentos a entrevista a profundidade e as anotações de campo. Resultados: Identificaram-se as categorias: "Enfrentando-me com a realidade", "Cuidado integral, o que sempre tem-me ensinado", "O meu relacionamento com o outro", "Ensinando ao meu paciente e à sua família", e "A família, o pilar fundamental". Conclusões: A experiência expressada pelos alunos gerou expectativas inesperadas, aplicaram os conhecimentos necessários para prestar os melhores e individualizados cuidados. Em cada participante surgiu a sensibilidade o que permitiu-lhe situar-se no lugar do outro, compreendendo a impotência, frustração e perdida do controle. O processo de atenção de enfermagem é o esquema fundamental para prestar cuidado integral do paciente.

Palavras-chave: Educação em enfermagem, estudantes de enfermagem, enfermagem, cuidado.

\section{Introducción}

A nivel mundial los traumatismos son un problema de salud pública; más de cinco millones de personas mueren al año; el mayor índice corresponde a menores de 40 años del sexo masculino. Llama la atención que el trauma craneoencefálico (TCE) (1) es la principal causa de mortalidad en pacientes poli traumatizados (2), dejando secuelas físicas (3), emocionales y psicológicas (4), siendo además la primera causa de muerte violenta por accidente de tránsito. Se estima que alrededor de 1.5 millones de personas ingresan cada año a los servicios de urgencias, debido a traumatismo craneal, de los cuales 230.000 son hospitalizados y 50.000 mueren como consecuencia de este (5). Según la Organización Mundial de la Salud (OMS), cerca de 3.500 personas mueren a causa de accidentes en las carreteras, siendo los más vulnerables niños, ancianos, ciclistas y peatones; de igual forma, cada año millones de personas presentan heridas y diversas discapacidades.

Con el ánimo de disminuir estos índices, el gobierno ha promovido el fomento de medidas de seguridad como el uso del casco y cinturón; a la vez se han impartido nuevas reglas como prueba de alcoholemia y el control de velocidad (6).

En Colombia por cada 100.000 habitantes se reportan más de 200 casos por TCE con predominio en hombres, generando principalmente incapacidad neurológica. De acuerdo con los porcentajes, el $70 \%$ de los pacientes se recupera de dicho evento, el $9 \%$ fallece antes de llegar a una institución de salud, el 6\% muere estando hospitalizado y el 15\% queda con secuelas físicas, psicológicas y cognitivas, lo cual genera limitaciones en la vida laboral, social, familiar y por ende, deterioro en la calidad de vida (7). Para el año 2012 este politraumatismo se convirtió en el $65.5 \%$ de las 
lesiones mortales por accidente de tránsito, por lo que es considerado un problema de salud pública a nivel nacional, de ahí la importancia de la intervención del profesional de enfermería para disminuir las complicaciones y ofrecer un cuidado integral y de esta forma contribuir a las políticas públicas para lograr una atención en salud de calidad (7).

Se debe precisar, que algunos de los principales factores asociados con las lesiones y los accidentes mortales por TCE son la conducción de vehículos a altas velocidades, la elevada densidad peatonal, el aumento de vehículos circulantes en las zonas urbanas, el consumo de alcohol y el déficit en los diseños e infraestructura de los sistemas de transporte (8). El cuidado (9) a personas hospitalizadas con TCE se debe realizar bajo el conocimiento y reflexión del profesional de enfermería con el fin de calmar, disminuir y prevenir lesiones secundarias (10) a través de la valoración, diagnóstico, ejecución y evaluación que realiza el enfermero a su paciente, para brindar cuidado integral e individualizado (11-13) y de esta forma ser responsable de su actuar, protegiendo la vida y valores del sujeto de cuidado (14).

En el cuidado a pacientes con TCE (15), hay que promover la enseñanza y preparación familiar para brindar un cuidado de calidad y prevenir complicaciones. Además, por medio de la relación enfermera-paciente se genera confianza para lograr los objetivos propuestos. Las personas con TCE, pueden estar conscientes o inconscientes según su gravedad, lo importante es encontrar sentido a lo que hacen sin importar las consecuencias finales; el compromiso y la relación están orientados a entender el significado de la situación de salud de quien se cuida con el fin de realizar acciones apropiadas y pertinentes en cada situación particular (16).

Por lo tanto, los estudiantes en su rol de cuidadores, reconocerán que todos los seres humanos necesitan de cuidado hasta su fin (17); es por eso que la formación debe estar orientada a brindar cuidado integral (18) en diferentes situaciones y reconocer que los sentimientos, emociones, percepciones, huellas, efectos, conmociones y pasiones (10) pueden surgir en esta situación en particular. Sin embargo, el estudiante se encuentra en una realidad en la que se le dificulta reconocer qué es lo que necesita para su verdadero aprendizaje, por tanto, las oportunidades en un medio real como lo son las prácticas formativas que servirán de base para que el docente oriente en cada acto de cuidado (19). Se puede señalar, que las experiencias de cuidado de los estudiantes se convierten en un método de gran utilidad donde se describe de manera verbal o escrita una situación vivida a través de la interacción al cuidar a la persona; no solo con un fin científico, sino también para explorar vivencias y determinar la responsabilidad hacia el sujeto de cuidado (20). Asimismo, es una oportunidad para evaluar la capacidad que tiene el futuro profesional de tomar decisiones y resolver inquietudes, expresadas de manera coherente, lógica, que sin duda, son la forma de interpretar su propia historia. Finalmente, el docente se encargará de orientarlo en su modo de proceder para que cada vez sean más significativas las experiencias de cuidado (21) y se configuren en un mecanismo transformador y creativo de la práctica clínica.

El significado que otorga el estudiante a la vivencia genera aportes a la disciplina, puesto que son comunicadas las necesidades, problemas, valores, conocimientos, costumbres y formas de vivir $(22,23)$ con el fin de crear estrategias de intervención que generen autonomía y fortalecimiento en la práctica del futuro profesional (24).

\section{Objetivos}

\section{Objetivo General}

Describir la experiencia de los estudiantes de enfermería en el cuidado de pacientes hospitalizados con trauma craneoencefálico durante el primer semestre académico de 2017, en una institución de salud de tercer nivel de atención de Villavicencio, Colombia.

\section{Objetivos Específicos}

- Reconocer los elementos más significativos de la experiencia de cuidado a pacientes con trauma craneoencefálico.

- Contribuir al conocimiento científico a través de la narrativa con el fin de acercarse a la práctica formativa como fuente de conocimiento.

- Determinar fenómenos de salud que están presentes en la práctica de cuidado.

\section{Materiales y Método}

Se trata de una investigación cualitativa de carácter fenomenológico. La fenomenología según Schütz 
ISSN-PRINT

1794-9831

E-ISSN 2322-7028

Vol. 16 No. 3

Sep - Dic 2019

Cúcuta, Colombia
(25) plantea que el sujeto realiza acciones que están cargadas de significados y estas acciones tienen un sentido; aunque el actor no haya tenido intención de significar algo, su acción puede ser interpretada por otro. El muestreo se realizó por conveniencia, pertinencia, adecuación, oportunidad y disponibilidad (26); para la selección de los participantes se acordó una reunión con los 27 estudiantes de VII semestre del Programa de Enfermería, quienes en el curso denominado Cuidado al Adulto I realizaron prácticas con pacientes hospitalizados de TCE en el primer semestre de 2017. En la reunión se explicó el objetivo de la investigación y la garantía de la privacidad de la información suministrada; posteriormente 12 estudiantes mujeres entre 20 y 25 años aceptaron participar y firmaron consentimiento informado. Los instrumentos utilizados fueron la entrevista a profundidad y el diario de campo (26), con el fin de esclarecer la experiencia humana subjetiva. Se realizaron de 3 a 4 entrevistas por participante, con una duración de 30 a 60 minutos. El primer encuentro partió de la pregunta central: “¿Qué significó para usted cuidar a un paciente con TCE?"; a partir de la respuesta se iban generando nuevas preguntas, teniendo en cuenta los sentimientos y emociones generados en cada experiencia de cuidado.

Con el fin de aplicar los criterios de fiabilidad y validez, las autoras del trabajo y un asesor externo realizaron la triangulación de la información (27) durante la fase inicial de la codificación mediante su confirmación de manera independiente. Se revisó la coincidencia de los testimonios, y cuando fue necesario se profundizó en las respuestas, sin omitir frases ni expresiones dentro de su contexto que luego se compararon con la grabación realizada. Posteriormente se leyó línea por línea, se establecieron descriptores los cuales se agruparon en códigos y luego en categorías que describían la experiencia. Algunas expresiones no verbales (gesticulaciones del rostro, movimientos de las manos, cambios en el estado de ánimo) fueron detalladamente observadas y registradas en el diario de campo. Los resultados del análisis de la información fueron puestos a consideración de los participantes con el fin de verificar la idoneidad, garantizando los criterios de credibilidad y confiabilidad (26). Cabe destacar que los participantes mantuvieron el interés durante el desarrollo del estudio. Se emplearon códigos para identificar la información aportada por cada uno.
La investigación fue realizada en el marco del convenio de relación docencia servicio, aprobado y avalado por el comité de bioética de la Universidad de los Llanos. Desde el inicio de la investigación se tuvieron en cuenta los aspectos éticos contemplados en la Resolución No 008430 del Ministerio de Salud de Colombia.

\section{Resultados}

La experiencia transcurre en el servicio de urgencias de una institución de salud de tercer nivel, en la práctica de séptimo semestre de enfermería. Durante el turno, los estudiantes participaron del cuidado a la persona con TCE, obteniendo diferentes significados. A continuación, se presentan las categorías que surgen del análisis de contenido.

\section{Me enfrento con la realidad}

Brindar cuidado a una persona con TCE, requiere de una atención delicada, son personas que fácilmente cambian de temperamento y que pueden tener comportamientos agresivos, por eso el estudiante siente temor e inquietud al verse enfrentado a la persona, sujeto de cuidado, a pesar de poseer los conocimientos para brindar la atención de enfermería.

"Estaba preocupada, no sabía cómo iniciar mi atención” [E2P3]. "[...] no sabía qué hacer, pues no tenía la experiencia de iniciar mi plan" [E1P4]. "Tenía mucha angustia, en realidad no sabía cómo cuidar a mi paciente" [E1P5]. "Sentí que no interactúe mucho con él... no pude aplicar mi plan de atención, no estaba preparada" [E1P6]. "No sabía qué hacer, la profesora me dijo, ¿ya inicio su plan de atención...?” [E2P8]. “...Mi mente pensaba solo en las dificultades y dije, pero ¿qué hago?, ¿por qué no realizo mi plan de atención comome lo enseñaron?" [E2P10]. "Los detalles pequeños, son de grandes significados, esa es la realidad... eso es cuidar" [E2P2].

\section{Cuidado integral, es lo que siempre me han inculcado}

La formación del profesional de enfermería está orientada a brindar cuidado integral que le permita realizar diferentes estrategias de intervención, para ver en cada ser humano una persona única en todas sus dimensiones, por tanto, se le acepta tal como es y se dignifica. 
Mi paciente es único “...dejar de pensar que son personas malas y estigmatizar solo porque no están bien vestidos...” [E3P1]. “... es atenderlo en todas sus dimensiones, sin importar su condición o conducta" [E2P3]. "Como enfermeras debemos ser más humildes..." E3P7. [...] "no estaba recibiendo una atención digna..." E3P9. “... el personal de salud se había familiarizado con él..., creando un lazo afectivo" [E3P10]. “... así sentí que le brindaba cuidado integral, sin pensar en cuidar una patología, sino a una persona” [E3P11]. “... ver lo que sentía su familia, eso me conmovió mucho". [E2P8]. [...] "el ser humano es un ser íntegro, el cual tiene unas necesidades específicas que dependen de múltiples factores..." [E2P12]. “... no solo se cuida a la persona hospitalizada sino también a su entorno..." [E3P11]. [...] "le preocupaba más la situación en la que se encontraba su hija pequeña, por eso le ayudé a saber cómo estaba ella” [E4P5]. [...] “... más allá de verlo con su trauma craneoencefálico, es apreciarlo como un ser holístico" [E3P1].

Un cuidado sin barrera "Me queda un sin sabor, pero sé que ejercí un cuidado humanizado y sin barreras y ayudé en su recuperación" [E4P1]. "En la práctica diaria se puede comprobar la falta de humanización... poco calor humano de los profesionales, y es donde juega nuestro actuar" [E3P5]. "Estoy segura de que aporté con las mejores estrategias para hacer del cuidado algo más agradable, de esta forma pensé en el cuidado integral y humanizado y ¿por qué no desde ahora?" [E2P6]. "Me despedí y le dije varias cosas sin la sensación de sentirme extraña, lo hice como si me estuviera mirando y escuchando, es decir, brindando un cuidado compasivo, competente y humanizado" [E2P8]. “... mi experiencia fue poco satisfactoria, la atención que se presta en los servicios de urgencias no logra las metas en humanización" [E1P10]. "El propósito de nuestra profesión, es brindar cuidado integral, no solo realizar procedimientos, por eso no es cualquier tipo de cuidado sino uno lleno de humanización y amor por el prójimo" [E3P11]. "La gran mayoría de ellos no logra tener una atención personalizada y humanizada, dejando atrás uno de los principios de la enfermería en el que se basa el cuidado humano" [E4P12]. "Le decía que todo iba a estar bien, pensando que él en este momento necesitaba de unas palabras de aliento, más que una inyección" [E4P3]. "El cuidado de enfermería siempre debe estar destinado a preservar el bienestar del paciente con la mayor calidez humana" [E2P10]. “... muchas veces la calidez hace el mejor papel de calmante y seguridad en los pacientes conscientes, y aunque no lo creamos en los inconscientes también..." [E3P7]. "Para mí fue extraño hablarle a alguien que no me veía y no me respondía, pensé que él necesitaba de mi cuidado humano" [E3P1]. “... se hace necesario que uno sea más humano todavía, se debe tener el doble de humanidad, la de uno y la de aquel que la está perdiendo" [E3P2].

Poca experiencia, pero un cuidado de calidad. "... yo con mi poca experiencia y conocimiento les expliqué esos interrogantes” [E2P4]. “... gracias a nuestros conocimientos es como podemos ofrecerles cuidados de calidad" [E3P3]. "Aporté un poco con mis conocimientos al cuidado integral del paciente" [E2P4]. "Empecé a retomar todo lo visto en el transcurso de mi carrera y a recordar la esencia de nuestra profesión" [E3P9]. "... lo que hice fue contar con mis conocimientos para brindar cuidado integral, a pesar de no tener mucha experiencia" [E3P12]. "A pesar de que tenía conocimiento de hacerlo, es muy difícil brindar cuidado" [E3AP2]. “... sentí inseguridad, no sé, tal vez, por la falta de experiencia, pero sabía que era muy importante mi atención de manera organizada" [E3P5]. "Creemos que la persona requiere cuidados extraordinarios que ni siquiera podemos identificar a simple vista, pero estamos muy equivocados" [E2P8]. “... el cuidado de enfermería va desde lo más complejo hasta lo más simple, puede ser brindar un vaso de agua o tan solo escuchar..." [E3P9].

\section{Mi relación con el otro}

Todo acto de cuidado inicia con una buena interrelación enfermera-paciente, por lo que es importante educar al estudiante de enfermería en este aspecto y demostrar que mediante esta interrelación se consiguen mejores resultados.

"Me pareció importante el respeto y la escucha activa, poco a poco fui ganando su confianza" [E4P2]. “... fue la oportunidad de conocer a cada
E-ISSN 2322-7028

Vol. 16 No. 3

Sep - Dic 2019

Cúcuta, Colombia 
ISSN-PRINT

1794-9831

E-ISSN 2322-7028

Vol. 16 No. 3

Sep - Dic 2019

Cúcuta, Colombia paciente y hacer un concepto propio, sin llegar a juzgar" [E3P3]. “... poco a poco la relación con mi paciente fue creciendo, me agradaba conversar con ella y expresarle mis sentimientos" [E3P4]. "Tan pronto inicié a conversar con él, cambio su rostro" [E2P8]. “... me dijo: sólo acompáñeme por favor un rato, en ese momento entendí que su necesidad era escucharlo" [E3P9]. “... al final recibí un fuerte abrazo de la paciente y sentí que a pesar de los sucesos cumplí mi labor". [E1P7]. “... me di cuenta por el lenguaje no verbal que lo que necesitaba era una llamada" [E1P5].

\section{Enseñándole a mi paciente y su familia}

Cada acto de cuidado es una oportunidad para brindar educación al paciente y su familia; es un espacio que se debe aprovechar y más aún con los pacientes con TCE, por las complicaciones y secuelas que deja la enfermedad.

“... la labor de la enfermería debe llegar a brindar los cuidados mediante la educación antes de dar de alta al paciente" [E4P6]. “... le expliqué lo que estaba sucediendo, dándole educación de cómo realizar sus cuidados" [E3P5]. "Aprendí la importancia de brindarle una buena educación, en cada momento que estuve a su lado" [E2P7]. " ... me di cuenta de que tenía muchas dudas acerca de su enfermedad, y cómo sería el cuidado en casa, por lo que inicié con mis explicaciones" [E4P1]. "... le daba las indicaciones a la familia sobre los cuidados, además de las precauciones y complicaciones" [E3IP2].

\section{La Familia, pilar fundamental}

La familia ocupa un lugar importante en el paciente hospitalizado, se convierte en apoyo y soporte para la persona con TCE; ante esta situación, se deben modificar las rutinas y aceptar las nuevas condiciones de vida que tendrá el paciente. La familia está ahí con su presencia siempre dispuesta a brindar bienestar y a posibilitar las medidas que estén a su alcance para mejorar y sobrellevar la condición de salud del paciente.

"Creo que la familia juega un papel primordial en la recuperación de estos pacientes" [E2P8]. "Su familia lo visitaba constantemente, esa voz de aliento que le daban ellos era su mejor medicina"
[E1P10]. "Podía evidenciar que la compañía de su familia lo motivaba para sentirse mejor" [E2P12]. "Busqué la oportunidad de hablar con su esposa y hermana, traté de tranquilizarlas” [E1P7]. “... y la importancia de integrar en este cuento a la familia, el impacto no solo es de quien se encuentra en la habitación, sino también del entorno familiar" [E1P5]. “... pero más allá de eso, pensar en su contexto familiar, son las personas que más deben brindar apoyo...". [E4P4].

\section{Discusión}

En la investigación se evidenció que la práctica formativa es un espacio propicio para que los estudiantes no solo afirmen sus conocimientos, sino también para que interactúen con el sujeto de cuidado e involucren sus emociones y sentimientos en cada oportunidad que se presenta. Desde el aula de clase, el estudiante comienza a conocer el significado de cuidar, considerado como una actitud de servicio para apoyar, ayudar y proteger a quienes están en una condición especial.

La categoría "Me enfrento a la Realidad", representa un sinnúmero de emociones por las que pasa el estudiante de enfermería, al experimentar la incertidumbre frente a una realidad palpable, en la cual debe aplicar lo que ha aprendido en su proceso de formación. Olivé et al. (28) afirman que, al momento de enfrentarse a la práctica formativa, el estudiante duda de sus conocimientos, siente miedo a hacer mal las cosas y se alteran sus signos vitales. Sin embargo, al finalizar la jornada los sentimientos se apaciguan, demostrando un grado de autoestima, al afirmar que el día siguiente le irá mejor con su plan de cuidado.

Por otra parte, el estudiante al enfrentarse al paciente con TCE hace que el futuro profesional de enfermería apoyado en sus conocimientos, fortalezca la atención y aplique los conceptos teóricos dentro del contexto real, por tanto, reconoce que su actuar es un factor determinante en la supervivencia y prevención de las secuelas en la persona con TCE $(11,29)$.

Como se demostró en este estudio, es necesario enfatizar que durante la formación de profesionales de enfermería, se debe tener en cuenta el cuidado holístico. La literatura señala que ésta forma de cuidado se inicia desde que se establece la relación enfermera-paciente, momento en el que se valora a la persona y su am- 
biente, teniendo en cuenta su vida y transcurrir, como ser biopsicosocial y espiritual y mantenerse tal como es (30). Al respecto, se mencionan los momentos que hacen parte de una buena interacción hasta lograr el afecto hacia el sujeto de cuidado: el primero hace referencia a la concientización, seguido por la creatividad, servicio en el cuidado, y finalmente la familiaridad con el otro; si se logra poner en práctica estos elementos, se estará fortaleciendo el cuidado holístico (31).

Por su parte, Damkliang et al. (32), desarrollaron una guía para pacientes con TCE, con base en la evidencia encontraron que su aplicación proporcionó grandes beneficios, no solo para quienes presentan esta enfermedad, sino también para los profesionales, puesto que incluía intervenciones de enfermería específicas que anteriormente no eran tenidas en cuenta por el personal, lo cual generó un gran aporte al cuidado integral de la persona con TCE.

De acuerdo a lo anterior, es necesario promover en el profesional de enfermería la conciencia (33) de utilizar marcos que permitan una descripción integral, holística y contextual de los fenómenos de la práctica para mejorar la salud y bienestar de los individuos (12). En la presente investigación se observa que los conceptos cuidado holístico y humanización, están implícitos y transcendieron en el estudiante, al tener en cuenta las necesidades individuales de los pacientes con TCE.

Es preciso aclarar que la atención humanizada es un proceso de sensibilización de las personas que lo ofrecen para hacer el bien, movidos por sentimientos de compasión, solidaridad y respeto hacia el ser humano; desafortunadamente, no se da muchas veces por la carga laboral del personal de enfermería y un sin número de funciones administrativas asignadas; sin embargo, es un elemento esencial que indica la calidad en el servicio prestado (18). En este estudio, los estudiantes fortalecieron la condición humana tanto del paciente como de sí mismos, volviéndose sensibles y responsables frente a las necesidades del otro.

Respecto a la relación del futuro profesional con la persona sujeto de cuidado, se pudo constatar que la comunicación por medio de palabras, gestos y actitudes dejan una impresión en quien se cuida, por tanto, se debe alcanzar la empatía necesaria para ayudar a resolver lo que el paciente necesita $(18,34)$. Como se evidencia en los testimonios de los participantes, esta relación fue única, expresada por la confianza, la presencia auténtica y la escucha; por tanto, si se acepta la situación del otro, se percibe su vulnerabilidad.

Lo anterior demuestra que una interrelación efectiva puede disminuir el dolor, el estrés, generar mayor comodidad, aceptación del tratamiento y facilitar una recuperación pronta (35). La comunicación enfermero-paciente es propicia para demostrar el respeto e interés por quien habla y escuchar de manera asertiva, dando una voz de aliento (20).

Debe señalarse, que el estudiante de enfermería experimenta diferentes sensaciones ante el paciente con TCE y por tanto necesita priorizar su verdadera necesidad de cuidado. Staskova y Tóthova (36) se refieren a la importancia de impartir simpatía en el cuidado como primer acto, aunque esta cualidad es algo que nace en cada persona, más aún en el enfermero, por lo que es indispensable que en la práctica formativa se prioricen las necesidades de cuidado (37); por ejemplo, permitir que el paciente sea visitado en horas diferentes a las asignadas, proporcionar una llamada, dar un café, agua o cualquier otro acto, siempre y cuando no cause daño a la persona (38).

A través de la educación, la persona sujeto de cuidado y su familia, conocen y comprenden la enfermedad y su proceso $(39,40)$; además genera cambios de comportamiento que contribuyen en el autocuidado del paciente durante la hospitalización y después del egreso (41); además sirve para mejorar las condiciones de la enfermedad que padece (42). Con la investigación, los estudiantes establecieron confianza y compromiso con el paciente y su familia, siendo capaces de educarlos; también, fortalecieron sus conocimientos y relaciones interpersonales.

Con respecto a la familia como pilar fundamental, se considera que es un actor significativo en el cuidado de la persona con TCE, por tanto, es importante crear confianza entre el estudiante, la familia y el paciente para mantener una relación acertada de cuidado y una mejor y pronta recuperación (27). Con su participación se establece una buena interacción familiar, pese al estado de salud o situación grave en que se encuentre el paciente (22).

Por otra parte, la familia es el agente principal de la recuperación prolongada de la persona con trauma, de su adaptación y discapacidad posterior (43). Al respecto, Sánchez (44) indica que el TCE no solo tie- 
ISSN-PRINT

1794-9831

E-ISSN 2322-7028

Vol. 16 No. 3

Sep - Dic 2019

Cúcuta, Colombia ne impacto en la persona que lo padece, sino en el entorno familiar, porque cambian las relaciones entre ellos, los roles que cada uno desempeña y las expectativas y planes a futuro.

Finalmente, es importante resaltar que el primer contacto del estudiante con el familiar del paciente, está lleno de temores enfocados a establecer una relación de confianza, sobre todo con personas que se encuentran gravemente enfermas; es frecuente que los pacientes y familiares sean quienes permitan brindarle al futuro profesional la oportunidad de entrar en sus vidas para proporcionar sus cuidados (29).

\section{Conclusiones}

- La experiencia expresada por los estudiantes en la atención del paciente hospitalizado con trauma craneoencefálico generó expectativas para las cuales no se encontraban preparados; sin embargo, lograron aplicar los conocimientos recibidos en el aula de clase, a fin de brindar el cuidado de manera individual, según las necesidades de la persona y su entorno.
- Los significados conferidos a la experiencia de cuidar al paciente con TCE, reflejan situaciones inesperadas para el estudiante, manifestándose la subjetividad por medio de diferentes expresiones, comprendiendo todo lo relacionado con el ser humano integral y holístico. Es por eso, que emergen sentimientos, emociones, aptitudes e inseguridades, producto de la práctica formativa en la que no se tiene en cuenta estos factores psicológicos.

- El estudiante permitió aflorar su sensibilidad, logrando situarse en la posición del otro (su paciente), entendiendo la impotencia, frustración y pérdida de control, lo que permitió que el paciente no se sintiera que estaba solo, al contrario, tenía en el futuro profesional un apoyo, una frase de aliento y la motivación de seguir viviendo, pese a las circunstancias.

\section{Conflicto de intereses}

Las autoras declaran no tener ningún conflicto de intereses.

\section{Referencias bibliográficas}

1. Moscote Salazar LR, Rubiano AM, Alvis Miranda HR, Calderón Miranda W, Alcalá Cerra G, et al. Severe Cranioencephalic Trauma: Prehospital Care, Surgical Management and Multimodal Monitoring. Bull Emerg Trauma [Internet]. 2016 [Consultado 13 enero 2018]; 4(1):8-23. Disponible en: https:// www.ncbi.nlm.nih.gov/pmc/articles/PMC4779465/

2. Piña A. Manejo del trauma craneoencefálico en la atención primaria en salud. MéD. UIS. [Internet]. 2015 [Consultado 15 abril 2017]; 28(1):153-158. Disponible en: https://dialnet.unirioja.es/descarga/ articulo/5168238.pdf

3. Calvette LF, Joanette Y, Fonseca RP. Traumatismo cranioencefálico: avaliação da ocorrência de heminegligência e de déficit atencional por tarefas de cancelamento. Avances en Psicología Latinoamericana. [Internet]. 2013 [Consultado 13 enero 2018]; 31(1):121-130. Disponible en: http://www. scielo.org.co/pdf/apl/v31n1/v31n1a10.pdf

4. Díaz Sosa DM, Arango Lasprilla JC, Espinosa Jove IG, Rivera Ledesma A. Necesidades y sobrecarga en cuidadores primarios informales de pacientes con traumatismo craneoencefálico. Psicología desde el Caribe. [Internet]. 2014 [Consultado 12 enero 2018]; 31(3):393-415. Disponible en: http://www. scielo.org.co/pdf/psdc/v31n3/v31n3a02.pdf

5. Sosa M, López J. Prevalencia del traumatismo craneoencefálico en una unidad médica de alta especialidad de la ciudad de Monterrey Nuevo León. Rev. Esc. Med. Dr. J. Sierra [Internet]. 2015 [Consultado 9 abril 2017]; 29(2):79-84. Disponible en: http://www.une.edu.mx/Resources/RevistaMedicina/2015/ Vol29No2.pdf

6. Organización Mundial de la Salud. Informe sobre la situación mundial de la seguridad vial. 19 de octubre de 2015. [Internet]. 2015 [Consultado 11 mayo 2016]. Disponible en: https://www.who.int/ violence injury prevention/road_safety_status/2015/es/ 
7. Ministerio de Salud y Protección Social. Guía de Práctica Clínica para el diagnóstico y tratamiento de pacientes adultos con trauma craneoencefálico. Sistema General de Seguridad Social en Salud [Internet]. 2014. [Consultado 13 de mayo de 2018]. Disponible en: http://gpc.minsalud.gov.co/gpc_sites/ Repositorio/Conv 563/GPC trauma craneo/GUIA\%20 COMPLETA TCE MEDITECH.pdf

8. Rodríguez JM, Camelo FA, Chaparro PE. Seguridad vial en Colombia en la década de la seguridad vial: resultados parciales 2010-2015. Rev Univ Ind Santander Salud. [Internet]. 2017 [Consultado 21 enero 2017]; 49(2):280-289. Disponible en: http://dx.doi.org/10.18273/revsal.v49n2-2017001

9. Carrera Quinteros MP. Atención de Enfermería a Pacientes con Trauma Craneoencefálico Grave que acuden al servicio de emergencia Hospital Pablo Arturo Suarez y propuesta de un protocolo de atención de enfermería, periodo de enero a diciembre 2012. Universidad de Guayaquil. [Tesis en internet]. 2015 [Consultado 29 octubre 2017]. Disponible en: http://repositorio.ug.edu.ec/handle/redug/9746

10. Henao Castaño M, Fajardo Ramos E, Núñez Rodríguez ML. La experiencia de estudiantes de enfermería ante el dolor infligido en la práctica clínica. Rev Salud Uninorte. 2014; 30(3):1-12.

11. Ydrovo Naranjo LA, Ruiz Coronel AP. Aplicación del proceso de atención de enfermería pacientes con traumatismo craneoencefálico por accidente de tránsito que ingresan a emergencia del hospital Roberto Gilbert Elizalde. Universidad de Guayaquil. [Internet]. 2015 [Consultado 29 octubre 2017]. Disponible en: http://repositorio.ug.edu.ec/bitstream/redug/9116/1/TESIS\%20FINAL\%20LINDAAZUCENA.pdf

12. Nairn W. Holistic care should be coming your way. Br Dent J. [Internet]. 2017 [Consultado 30 octubre 2017]; 223(8):568-569. Disponible en: https://doi.org/10.1038/sj.bdj.2017.878

13. Saca Naula BM, Sichiqui Ochoa DA, Quito Saca CF. Cuidado de enfermería a pacientes con traumatismo craneoencefálico del área de cirugía del Hospital "Vicente Corral Moscoso", Cuenca [Internet]. 2013 [Consultado 29 octubre 2017]. Disponible en: http://dspace.ucuenca.edu.ec/handle/123456789/4868

14. Damakliang J, Considine J, Kent B, Street M. Nurses'perceptions perceptions of using an evidencebased care bundle for initial emergency nursingmanagement of patients with severe traumatic brain njury: A qualitative study. Int Emerg Nurs [Internet]. 2015 [Consultado 6 octubre 2017]; 17(4):299305. Disponible en: http://www.internationalemergencynursing.com/article/S1755-599X(15)00046-4/ fulltext

15. Jaimes LM, Thompson HJ, Landis CA, Warms CA. Nurses' Knowledge management for patients with combat-related traumatic brain injuries on rehabilitation units. Rehabil Nurs [Internet]. 2015 [Consultado 30 octubre 2017]; 40(2):74-83. Disponible: http://onlinelibrary.wiley.com/doi/10.1002/ rnj.156/abstract;jsessionid=27B62EACEDA84F4C10F300633FFF101E.f03t03

16. Newman MA, Sime AM, Corcoran Perry SA. The focus of the discipline of nursing. ANS Adv Nurs Sci [Internet]. 1991 [Consultado 14 enero 2017]; 14(1):1-6. Disponible en: https://www.ncbi.nlm.nih. gov/pubmed/1819254

17. Edo Gual M, Monforte Royo C, Tomas Sábado J. Afrontar el sufrimiento y la muerte: desafíos para el cuidado en el siglo XXI. Enferm Clin. 2014; 25(1):42-43.

18. Beltran Salazar OA. Humanized care: A relationship of familiarity and affectivity. Invest. educ. enferm [Internet]. 2015 [Consultado 3 septiembre 2017]; 33(1):17-27. Disponible en: http://www.scielo. org.co/scielo.php?script $=$ sci arttext\&pid $=\mathrm{S} 0120-53072015000100003 \& \operatorname{lng}=\mathrm{en} \& \mathrm{nrm}=\mathrm{iso}$

19. Rivera Alvarez L. Saber y experiencias de el/la estudiante de enfermería en sus prácticas de cuidado. [Tesis Doctoral] 2013. [Consultado 31 mayo 2017]. Disponible en: http://www.tdx.cat/bitstream/handle/10803/132808/01.LNRA_TESIS.pdf;sequence=1

20. Uribe Velásquez SP, Lagoueyte Gòmez MI. "Estar ahi”, Significado del cuidado espiritual: La mirada de los profesionales de enfermería. Avances en Enfermería [Internet]. 2014 [consultado 11 octubre 2017]; 32(2):261-270. Disponible en: https://doi.org/10.15446/av.enferm.v32n2.46230

21. Muñoz Pino IP. Experience of nursing students upon their first care encounter with terminally ill pa- 
ISSN-PRINT

$1794-9831$

E-ISSN 2322-7028

Vol. 16 No. 3

Sep - Dic 2019

Cúcuta, Colombia

tients. Invest Educ Enferm. 2014;32(1): 87-94.

22. Briñez K. Narrativa de enfermería: visión y patrones de conocimiento en una entrevista en el hogar. Revista Colombiana de enfermería. 2015; 9(9):142-148.

23. Brint S. Obligated to Care: A Personal Narrative of Compassion Fatigue in an Oncology Nurse. J Holist Nurs. [Internet]. 2017 [Consultado 11 mayo 2018]. 35(3):296-309. Disponible en: https://www. ncbi.nlm.nih.gov/pubmed/27582127.

24. Gómez Ramírez OJ, Gutiérrez E. La situación de enfermería fuente de conocimiento de enfermería la narrativa como medio para comunicarla. Bogotá: Universidad nacional de Colombia, Bogotá, D.C.; 2011.

25. Schütz A. La construcción significativa del mundo social. Introducción a la sociología comprensiva. Barcelona: Ediciones Paidós; 1932. 1a reimpresión en España, 1993.

26. Hernández Sampieri R, Fernández Collado C, Baptista Lucio P. Metodología de la Investigación. 5 ed. 2010 [Libro en internet]. [Consultado 11 mayo 2016]. Disponible en: https://www.esup.edu. pe/descargas/dep investigacion/Metodologia $\% 20 \mathrm{de} \% 201 \mathrm{a} \% 20$ investigaci $\% \mathrm{C} 3 \% \mathrm{~B} 3 \mathrm{n} \% 205 \mathrm{ta} \% 20$ Edici\%C3\%B3n.pdf

27. Noreña AL, Alcaraz Moreno N, Rojas JG, Rebolledo Malpica D. Aplicabilidad de los criterios de rigor y éticos en la investigación cualitativa. Aquichan. 2012;12(3):263-74.

28. Olivé Ferrer MC, Getino Canseco M, Sanfeliu Cortés V, Bardají Fandos T. El diario reflexivo. Una vivencia de aprendizaje en las estancias clínicas enfermeras. Revista Iberoamericana de Educación [Internet]. 2014 [Consultado 22 abril 2018]; 65(2):1-13 Disponible en: http://diposit.ub.edu/dspace/ bitstream/2445/56473/1/642787.pdf

29. Jara Sanabria F, Lizano Pérez A. Aplicación del proceso de atención de enfermería por estudiantes, un estudio desde la experiencia vivida. Enfermería universitaria. [Internet]. 2016 [Consultado 22 abril 2018]; 13(4):208-215. Disponible en: http://dx.doi.org/10.1016/j.reu.2016.08.003

30. Meleis AI. Theoretical Nursing: Development and Progress. 5th. Ed. Philadelphia: Lippincott Williams \& Wilkins. Capítulo 5; 2012.

31. Medeiros RMK, Teixeira RC, Nicolini AB, Alvares AS, Corrêa ACP, Martins DP. Humanized Care: insertion of obstetric nurses in a teaching hospital. Rev Bras Enferm [Internet]. 2016 [Consultado 22 abril 2018]; 69(6):1029-36. Disponible en: http://dx.doi.org/10.1590/0034-7167-2016-0295

32. Damkliang J, Considine J, Kent B, Street M. Initial emergency management of patients with severe traumatic brain injury: development of an evidence-based care bundle for the Thai emergency department context. Australas Emerg Nurs J. [Internet]. 2014 [consultado 2018 abr 22]; 17(4):152-60. Disponible en: http://dx.doi.org/10.1016/j.aenj.2014.05.005

33. Albaqawi HM, Butcon VR, Molina RR. Awareness of holistic care practices by intensive care nurses in north-western Saudi Arabia. Saudi Med j [Internet]. 2017 [Consultado 30 octubre 2017]; 38(8):826831. Disponible en: https://www.ncbi.nlm.nih.gov/pmc/articles/PMC5556299/

34. Calegari R, Massarollo MC, Santos MJ. Humanização da assistência à saúde na percepção de enfermeiros e médicos de um hospital privado. Rev. esc. enferm. USP [Internet]. 2015 [Consultado 3 octubre 2017]; 49(2):42-47. Disponible en: http://dx.doi.org/10.1590/S0080-623420150000800006

35. Mena Gómez I, Troncoso Pérez D, González Ortega Y. Las teorías de enfermería y la relación enfermera paciente: aplicación para la práctica. Enfoque. Revista Científica de Enfermería [Internet]. 2014 [Consultado 2 noviembre 2015]; 15(10):17-20. Disponible en: http://www.repositoriodev.up.ac. pa/364/1/2\%20REVISTA $\% 20$ ENFOQUE $\% 20$ 10.pdf

36. Stasková V, Tóthová V. Conception of the human-to-human relationship in Nursing. Kontakt [Internet]. 2015 [Consultado 6 septiembre 2017]; 17(4):184-189. Disponible en: https://doi.org/10.1016/j. kontakt.2015.09.002

37. Gómez Rojas ML, Rodríguez Díaz BL. Situación de enfermería como herramienta para enseñar el 
proceso de atención de enfermería. Rev Cuid [Internet]. 2013 [Consultado 10 agosto 2018]; 4(1):144149. Disponible en: https://doi.org/10.15649/cuidarte.v4i1.16

38. Uriarte $\mathrm{S}$, Ponce G, Bernal M. Vivencias Cotidianas en espacios clínicos del estudiante de enfermería. Enfermería Universitaria [Internet]. 2016 [Consultado 2 octubre 2017]; 13(3):171-177. Disponible en: doi.org/10.1016/j.reu.2016.07.002

39. Narayana S, Rajkva Rajkomar A, Harrison JD, Valencia V, Dhaliwal G, Ranji SR. What Happened to my patient an educational intervention to facilitate postdischarge patient ffollow-up. J Grand Med Educ [Internet]. 2017 [Consultado 2 octubre 2017]; 9(5):627-633. Disponible en: http://www.jgme. org/doi/10.4300/JGME-D-16-00846.1? code=gmed-site

40. Casanova Moreno MC, Bayarre Vea HD, Navarro Despaigne DA, Sanabria Ramos GT, Delgado M. Efectividad de una estrategia educativa dirigida a adultos mayores diabéticos tipo 2 y proveedores de salud. Rev Cubana Salud Pública [Internet]. 2017 [Consultado 17 junio 2017]; 43(3):1-17. Disponible en: http://scielo.sld.cu/scielo.php?script=sci_arttext\&pid=S0864-34662017000300003\&lng=es

41. Siudakn Z, Krawczyk Ożóg A, Twarda I, Franczak I, Rajtar Salwa R, et al. "Heart without smoke" educational campaign - the role of patient education in secondary prevention of cardiovascular disease. Kardiol Pol [Internet]. 2017 [Consultado 2 octubre 2017]; 76(1):125-129. Disponible en. https:// www.ncbi.nlm.nih.gov/pubmed/28832093

42. Diez Manglano J, Recio Iglesias J, Varela Aguilar JM, Almagro Mena P, Zubillaga Garmendia G. Effectiveness of a simple intervention on management of acute exacerbations of chronic obstructive pulmonary disease and its cardiovascular comorbidities: COREPOC study [Internet]. 2017 [Consultado 8 octubre 2018]; 149(6):240-247. Disponible en: https://www.ncbi.nlm.nih.gov/pubmed/28396131

43. Arias M, García Vivar C. The transition of palliative care from the hospital to the home: a narrative review of experiences of patients and family caretakers. Invest Educ Enferm [Internet]. 2015 [Consultado 28 septiembre 2018]; 33(3):482-491. Disponible en: https://doi.org/10.17533/udea.iee.v33n3a12

44. Sánchez Raja E. Salud Sexual y calidad de vida en personas con traumatismos craneoencefálicos moderados (TCEM) [Tesis Doctoral]. Barcelona: Universidad de Barcelona; 2014 [consultado 20 septiembre 2018]. Disponible en: http://www.tdx.cat/bitstream/handle/10803/298167/ESR_TESIS. pdf; sequence $=1$ 